\title{
Coulomb-Induced Positive Current-Current Correlations in Normal Conductors
}

\author{
Andrew M. Martin and Markus Büttiker \\ Département de Physique Théorique, Université de Genève, CH-1211 Genève 4, Switzerland
}

(Received 18 November 1999)

\begin{abstract}
In the white-noise limit current correlations measured at different contacts of a mesoscopic conductor are negative due to the antisymmetry of the wave function (Pauli principle). We show that current fluctuations at capacitive contacts induced via the long range Coulomb interaction due to charge fluctuations in the mesoscopic sample can be positively correlated. The positive correlations are a consequence of the extension of the wave functions into areas near both contacts. As an example we investigate in detail a quantum point contact in a high magnetic field under conditions in which transport is along an edge state.
\end{abstract}

PACS numbers: 72.10. $-\mathrm{d}, 72.70 .+\mathrm{m}$

Fluctuations in beams of photons have long been of interest in optics. In 1954 Hanbury Brown and Twiss [1] showed that an investigation of the correlations of light of a star permits the determination of the diameter of the star. Electrical analogs to this effect have long been suggested but due to the interaction between carriers, it is difficult to achieve the necessary degeneracy in a vacuum beam experiment. In electrical conductors, however, the situation is fundamentally different, since at low temperatures, high filling factors are easy to achieve. Current-current correlations in multiterminal conductors were analyzed theoretically using both quantum theories and classical approaches [2-5]. It can easily be demonstrated that in the white-noise limit, current-current correlations in phasecoherent conductors are negative [2,6]. Indeed, recent experiments by Henny et al. [7], Oliver et al. [8], and Oberholzer et al. [9] have demonstrated negative correlations in good agreement with theory. The fermionic character of current fluctuations demonstrated in these experiments reflects the independent quasiparticle transport in the low frequency white-noise limit [10]. However, such a simple situation cannot be expected to hold over a wide range of frequencies. It is well known that time-dependent transport in conductors is collective due to the long range Coulomb interaction, which leads to bosonic excitations (plasmons), and that consequently we expect a different (bosonic) behavior of current-current fluctuations. In this work, we demonstrate that already at (in principle) arbitrarily low frequencies it is possible to find current correlations which are positively correlated in purely normal conductors. To achieve this, we investigate current fluctuations and their correlations at contacts which are purely capacitively coupled to a conductor. Current at these contacts is a consequence of charge fluctuations in the mesoscopic conductor and not, as in the theories and experiments discussed above, a consequence of carriers transmitted or reflected into different contacts.

We initially consider the general system shown in Fig. 1, where we have a mesoscopic conductor in contact with two ideal leads ( 1 and 2 ) and Coulomb coupled to two macroscopic gates $(A$ and $B)$. We consider the scenario where the two gates are Coulomb coupled to separate regions of the conductor $\left(\Omega_{A}\right.$ and $\left.\Omega_{B}\right)$, and assume that there is no direct Coulomb coupling between regions $\Omega_{A}$ and $\Omega_{B}$. It will be apparent how such effects could be taken into account. Having shown how it is possible to calculate the charge correlations between the two gates $(A$ and $B)$ we proceed to consider four specific examples, for a quantum point contact (QPC) in a high magnetic field, demonstrating that for these systems the current correlations can be both positive and negative.

Within the context of scattering theory the charging and charge fluctuation phenomena in gated mesoscopic conductors have already been investigated $[11,12]$. The work described in this Letter develops this understanding for structures with more than one macroscopic gate coupled to the mesoscopic conductor. It has already been shown [12] that for a mesoscopic structure Coulomb coupled to a single macroscopic gate the fluctuations in the charge in the mesoscopic region induce charge fluctuations in a macroscopic gate which is placed in close proximity to the mesoscopic region. The dynamics of the system are

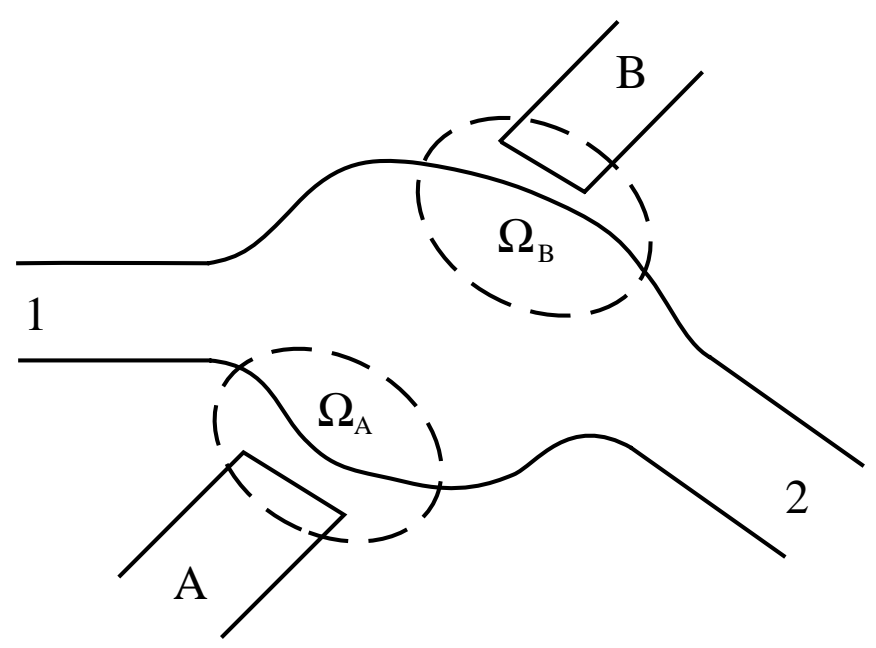

FIG. 1. Conductor connected via ideal leads (1 and 2$)$ to reservoirs with spatially separate regions capacitively coupled to gates $(A$ and $B)$. 
governed by two quantities, the charge relaxation resistance of the structure $R$ and the electrochemical capacitance of the structure $C_{\mu} . R C_{\mu}$, in analogy with macroscopic systems, denotes the charge relaxation time of the conductor. For a mesoscopic system, at equilibrium, Coulomb coupled to a single macroscopic gate, in the zero temperature limit, the current fluctuation spectrum in the gate, to leading order in the frequency, is given by [12] $S_{I I}^{q}(\omega)=2 \omega^{2} \hbar|\omega| C_{\mu}^{2} R_{q}$, where $R_{q}$ is the equilibrium charge relaxation resistance. Out of equilibrium and at zero temperature it has been shown to leading order in the applied voltage that [12] $S_{I I}^{V}(\omega)=2 \omega^{2}|e V| C_{\mu}^{2} R_{V}$, where we call $R_{V}$ the Schottky resistance to emphasize that the charge fluctuations are associated with shot noise. In the presence of more than one gate we show below that the equivalent spectrum is now given by

$$
\begin{aligned}
& S_{I_{\alpha} I_{\beta}}^{q}(\omega)=2 \omega^{2} \hbar|\omega| C_{\mu}^{\alpha} C_{\mu}^{\beta} R_{q}^{\alpha \beta}, \\
& S_{I_{\alpha} I_{\beta}}^{V}(\omega)=2 \omega^{2}|e V| C_{\mu}^{\alpha} C_{\mu}^{\beta} R_{V}^{\alpha \beta},
\end{aligned}
$$

where the subscripts and superscripts $\alpha$ and $\beta$ specify the gates in the system. For example, if $\alpha=\beta$ then Eq. (1) defines the equilibrium current fluctuation spectrum in gate $\alpha$, whereas if $\alpha \neq \beta$ Eq. (1) defines the equilibrium current correlations between gates $\alpha$ and $\beta$.

Consider a mesoscopic conductor attached to $L$ normal ideal leads. This conductor can be described by a scattering matrix, with elements $s_{\gamma \delta}$, which relates electron amplitudes incident from contact $\delta$ to outgoing amplitudes in contact $\gamma$. If we let regions of the mesoscopic conductor be capacitively coupled to gates, as depicted in Fig. 1, the current fluctuations induced in a given gate are related to the charge fluctuations in the region of the conductor which is Coulomb coupled to the gate. If we have, as in Fig. 1, more than one gate, then we need to calculate the charge fluctuations in separate regions of the conductor, allowing us to then calculate both the charge fluctuations and the correlations of the currents in the gates. The bare charge fluctuations in a region $\Omega_{\alpha}$ are governed by the density of states matrix [11], whose elements are

$$
\mathcal{N}_{\delta \gamma}^{(\eta)}(\boldsymbol{r})=\frac{-1}{2 \pi i} \sum_{\nu}\left[s_{\nu \delta}^{\star}(E, U(\boldsymbol{r})) \frac{\delta s_{\nu \gamma}(E, U(\boldsymbol{r}))}{e \delta U(\boldsymbol{r})}\right],
$$

where $\boldsymbol{r}$ is in the volume $\Omega_{\eta}$ and $U(\boldsymbol{r})$ is the electrostatic potential at position $\boldsymbol{r}$. For example, $\mathcal{N}_{12}^{(A)}(\boldsymbol{r})$ is the electron density, at position $\mathbf{r}$ in volume $\Omega_{A}$, associated with two electron current amplitudes incident from contacts 1 and 2. $\mathcal{N}_{\gamma \gamma}^{(\eta)}(\boldsymbol{r})$ are elements of a generalized local Wigner-Smith time-delay matrix [13]. The explicit relation of the charge operator to local wave functions is given in [11], and a detailed derivation is found in Ref. [14].

The fluctuations of the bare charge in a region $\Omega_{\eta}$ can be found from the charge operator [12] $e \hat{\mathcal{N}}_{(\eta)}$ given by

$$
\begin{aligned}
\hat{\mathcal{N}}_{\eta}(\omega)= & \sum_{\delta \gamma} \int_{\Omega_{\eta}} d^{3} \boldsymbol{r} \int d E \hat{a}_{\delta}^{\dagger}(E) \\
& \times \mathcal{N}_{\delta \gamma}^{(\eta)}(\boldsymbol{r} ; E, E+\hbar \omega) \hat{a}_{\gamma}(E+\hbar \omega),
\end{aligned}
$$

where the zero-frequency limit of $\mathcal{N}_{\delta \gamma}^{(\eta)}(\boldsymbol{r} ; E, E+\hbar \omega)$ is given by Eq. (3). In the above equation $\hat{a}_{\delta}^{\dagger}(E)$ creates an incoming electron in lead $\delta$. The true charge fluctuations must be obtained by taking into account the Coulomb interaction.

Given the above equations we now want to consider the system shown in Fig. 1 and to calculate charge fluctuations in the two regions $\Omega_{A}$ and $\Omega_{B}$, hence enabling us to deduce the charge in each of the two gates. The first step is to find an expression for the charge operator in the two regions. Assuming that the geometrical capacitances which couple the two gates to two separate regions of the mesoscopic conductor dominate all other capacitances we can express the charge in the two regions of the conductor in two ways. First with the help of the potential operators for the two regions, $\hat{U}_{A(B)}$, we have

$$
\begin{aligned}
& \hat{Q}_{A}=C_{A} \hat{U}_{A}+C\left(\hat{U}_{A}-\hat{U}_{B}\right), \\
& \hat{Q}_{B}=C_{B} \hat{U}_{B}+C\left(\hat{U}_{B}-\hat{U}_{A}\right),
\end{aligned}
$$

where $C$ is the capacitive coupling between regions $\Omega_{A}$ and $\Omega_{B}$. The two preceding equations assume that the gates $A$ and $B$ are macroscopic and have no dynamics of their own. We can also determine the charge in a region $\hat{Q}_{A(B)}$ as the sum of the bare charge fluctuations in the region of interest $e \hat{\mathcal{N}}_{A(B)}$ and the induced charges generated by a fluctuating induced electrical potential in the same region. Within the random phase approximation the induced charges are proportional to the average frequency dependent density of states,

$$
N_{A(B)}=\sum_{\gamma} \int_{\Omega_{A(B)}} d^{3} \boldsymbol{r} \mathcal{N}_{\gamma \gamma}^{(A(B))}(\boldsymbol{r}),
$$

in the region of interest times the fluctuating potential. Thus the net charge, in regions $A$ and $B$, is

$$
\begin{aligned}
& \hat{Q}_{A}=C_{A} \hat{U}_{A}+C\left(\hat{U}_{A}-\hat{U}_{B}\right)=e \hat{\mathcal{N}}_{A}-e^{2} N_{A} \hat{U}_{A}, \\
& \hat{Q}_{B}=C_{B} \hat{U}_{B}+C\left(\hat{U}_{B}-\hat{U}_{A}\right)=e \hat{\mathcal{N}}_{B}-e^{2} N_{B} \hat{U}_{B} .
\end{aligned}
$$

Solving Eqs. (8) and (9) gives us, for the potential operators,

$$
\left(\begin{array}{c}
\hat{U}_{A} \\
\hat{U}_{B}
\end{array}\right)=\left(\frac{1}{d}\right)\left(\begin{array}{cc}
{\left[G_{B}\right]^{-1}} & C \\
C & {\left[G_{A}\right]^{-1}}
\end{array}\right)\left(\begin{array}{c}
e \hat{\mathcal{N}}_{A} \\
e \hat{\mathcal{N}}_{B}
\end{array}\right),
$$

where $G_{\alpha}=\left[C_{\alpha}+C+e^{2} N_{\alpha}\right]^{-1}$ and $d=$ $\left[G_{A}\right]^{-1}\left[G_{B}\right]^{-1}-C^{2}$. Now that we have an expression for the potential operators we can calculate the fluctuation spectra of the internal potentials $2 \pi S_{U_{\alpha} U_{\beta}}(\omega) \delta(\omega+$ $\left.\omega^{\prime}\right)=(1 / 2)\left\langle\hat{U}_{\alpha}(\omega) \hat{U}_{\beta}\left(\omega^{\prime}\right)+\hat{U}_{\beta}\left(\omega^{\prime}\right) \hat{U}_{\alpha}(\omega)\right\rangle$, where $\langle\cdots\rangle$ denotes a quantum statistical average over products of $\hat{a}$ and $\hat{a}^{\dagger}$ operators [11]. From this and using the fact 
that $S_{Q_{\alpha} Q_{\beta}}=C_{\alpha} C_{\beta} S_{U_{\alpha} U_{\beta}}$ we obtain the spectra of the screened charges. For $C=0$ (i.e., no capacitive coupling between the two regions $\Omega_{A}$ and $\Omega_{B}$ ) we find

$$
S_{Q_{\alpha} Q_{\beta}}(\omega)=\frac{C_{\mu_{\alpha}} C_{\mu_{\beta}}}{N_{\alpha} N_{\beta}} \sum_{\gamma \delta} \int d E F_{\delta \gamma} \operatorname{Tr}\left[\mathcal{N}_{\delta \gamma}^{(\alpha)}\left(\mathcal{N}_{\delta \gamma}^{(\beta)}\right)^{\dagger}\right]
$$

where $\quad F_{\delta \gamma}=f_{\delta}(E)\left[1-f_{\gamma}(E+\hbar \omega)\right]+f_{\gamma}(E+$ $\hbar \omega)\left[1-f_{\delta}(E)\right]$,

$$
\mathcal{N}_{\delta \gamma}^{(\eta)}=\int_{\Omega_{\eta}} d^{3} \boldsymbol{r} \mathcal{N}_{\delta \gamma}^{(\eta)}(\boldsymbol{r})
$$

and the electrochemical capacitance for a region $\eta$ is

$$
C_{\mu_{\eta}}=\frac{e^{2} N_{\eta} C_{\eta}}{C_{\eta}+e^{2} N_{\eta}} .
$$

Equation (11) gives us an expression for the charge fluctuation spectra. When $\alpha=\beta$, we have the fluctuation spectra in the gate $\alpha$, and when $\alpha \neq \beta$, Eq. (11) gives us the charge correlations between the two gates.

At equilibrium, at zero temperature Eq. (11) can be simplified to $S_{Q_{\alpha} Q_{\beta}}(\omega)=2 C_{\mu_{\alpha}} C_{\mu_{\beta}} R_{q}^{\alpha \beta} \hbar|\omega|$ where

$$
R_{q}^{\alpha \beta}=\frac{h}{2 e^{2}} \frac{\sum_{\gamma \delta} \operatorname{Tr}\left[\mathcal{N}_{\delta \gamma}^{(\alpha)}\left(\mathcal{N}_{\delta \gamma}^{(\beta)}\right)^{\dagger}\right]}{\operatorname{Tr}\left[\sum_{\gamma} \mathcal{N}_{\gamma \gamma}^{(\alpha)}\right] \operatorname{Tr}\left[\sum_{\gamma} \mathcal{N}_{\gamma \gamma}^{(\beta)}\right]} .
$$

The other limit we can consider is the zero temperature, low frequency limit of the charge spectra to leading order in applied voltage. Evaluating Eq. (11) we find $S_{Q_{\alpha} Q_{\beta}}(\omega)=2 C_{\mu_{\alpha}} C_{\mu_{\beta}} R_{V}^{\alpha \beta}|e V|$ where

$$
R_{V}^{\alpha \beta}=\frac{h}{2 e^{2}} \frac{\operatorname{Tr}\left[\mathcal{N}_{12}^{(\alpha)}\left(\mathcal{N}_{12}^{(\beta)}\right)^{\dagger}\right]+\operatorname{Tr}\left[\mathcal{N}_{21}^{(\alpha)}\left(\mathcal{N}_{21}^{(\beta)}\right)^{\dagger}\right]}{\operatorname{Tr}\left[\sum_{\gamma} \mathcal{N}_{\gamma \gamma}^{(\alpha)}\right] \operatorname{Tr}\left[\sum_{\gamma} \mathcal{N}_{\gamma \gamma}^{(\beta)}\right]} .
$$

From $S_{I_{\alpha} I_{\beta}}=\omega^{2} S_{Q_{\alpha} Q_{\beta}}$ we obtain Eqs. (1) and (2).

Having constructed the above methodology we now wish to consider a simple example to see if the current correlations between gates are positive or negative. The example we choose is a QPC in a high magnetic field [15], such that the transport is governed by edge states. We consider four scenarios, as shown in Fig. 2, keeping gate $A$ fixed and calculating the charge correlations between gate $A$ and gate $B$ when gate $B$ is in each of the four separate positions shown. First we consider the situation where gate $A$ is adjacent to gate $B$ [in position (i)]. These two gates are Coulomb coupled to two separate regions of the same edge state of interest $\left(\Omega_{A}\right.$ and $\left.\Omega_{B}\right)$.

If we consider only one edge state, then the scattering matrix for this system is

$$
\mathbf{S}=\left(\begin{array}{cc}
r & t \\
t^{\prime} e^{\left(i \theta_{A}+i \theta_{B}\right)} & r^{\prime} e^{\left(i \theta_{A}+i \theta_{B}\right)}
\end{array}\right),
$$

where the phases, $\theta_{A}$ and $\theta_{B}$, have been introduced explicitly. They arise from the fact that a carrier traversing region $A$ or $B$ adjacent to either of the gates acquires a phase $\theta_{A}\left(U_{A}\right)$ or $\theta_{B}\left(U_{B}\right)$ depending upon the region over

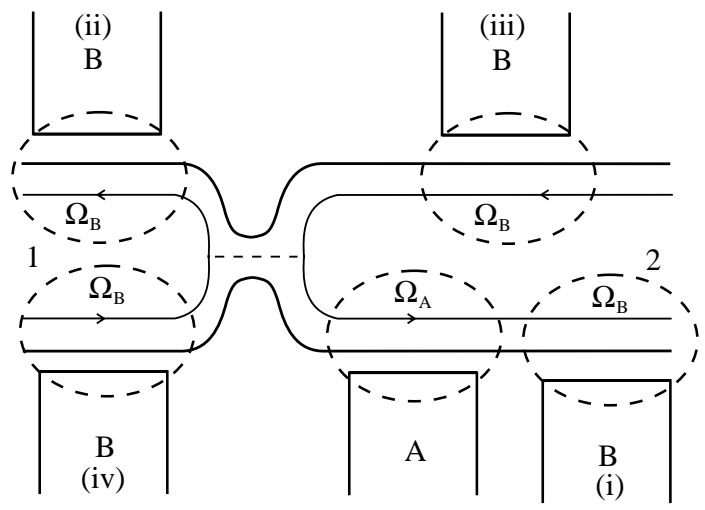

FIG. 2. QPC in a high magnetic field. Gate $A$ is fixed and the charge correlations between gates $A$ and $B$ for the four positions (i), (ii), (iii), and (iv) of gate $B$ are evaluated. In each case the two gates ( $A$ and $B$ ) are Coulomb coupled to the edge state which traverses regions $\Omega_{A}$ and $\Omega_{B}$.

which the carrier is traversing. $U_{A}\left(U_{B}\right)$ characterizes the potential in region $A(B)$.

To calculate the charge operator we have to evaluate the variation of the scattering matrix with respect to the potential $U_{A}$ and $U_{B}$ [Eq. (3)]. Only $s_{21}$ and $s_{22}$ depend upon these potentials. We find

$$
\frac{d s_{\delta \gamma}}{e d U_{A(B)}}=\left(\frac{d s_{\delta \gamma}}{d \theta_{A(B)}}\right)\left(\frac{d \theta_{A(B)}}{e d U_{A(B)}}\right) .
$$

But $\left(d \theta_{A(B)} / e d U_{A(B)}\right)=-2 \pi N_{A(B)}$ where $N_{A(B)}$ is the density of states of the edge state in region $\Omega_{A(B)}$. From this we find $\mathcal{N}_{11}^{(A(B))}=N_{A(B)} T, \mathcal{N}_{22}^{(A(B))}=N_{A(B)} R$, and

$$
\mathcal{N}_{12}^{(A(B))}=\left(\mathcal{N}_{21}^{(A(B))}\right)^{\star}=N_{A(B)}\left[\left(t^{\prime}\right)^{\star} r^{\prime}\right],
$$

where $T=|t|^{2}=\left|t^{\prime}\right|^{2}$ and $R=1-T$. Using the fact that the general scattering matrix for the system can be parametrized in the following manner:

$$
\mathbf{S}=\left(\begin{array}{cc}
i \sqrt{R} e^{i\left(\chi+\phi_{1}\right)} & \sqrt{T} e^{i\left(\chi+\phi_{2}\right)} \\
\sqrt{T} e^{i\left(\chi-\phi_{2}\right)} & i \sqrt{R} e^{i\left(\chi-\phi_{1}\right)}
\end{array}\right)
$$

we have for Eq. (16)

$$
\begin{aligned}
r & =i \sqrt{R} e^{i\left(\chi+\phi_{1}\right)}, & r^{\prime} & =i \sqrt{R} e^{i\left[\chi-\phi_{1}-\left(\theta_{A}+\theta_{B}\right)\right]}, \\
t & =\sqrt{T} e^{i\left(\chi+\phi_{2}\right)}, & t^{\prime} & =\sqrt{T} e^{i\left[\chi-\phi_{2}-\left(\theta_{A}+\theta_{B}\right)\right]} .
\end{aligned}
$$

Thus from the above we find

$$
R_{q}^{\alpha \beta}=\left(h / 2 e^{2}\right) \quad \text { and } \quad R_{V}^{\alpha \beta}=\left(h / e^{2}\right) T R .
$$

What we are really interested in is the sign of Eqs. (1) and (2); we know for this system that both $R_{q}^{\alpha \beta}$ and $R_{V}^{\alpha \beta}$ are positive, and $C_{\mu_{A}}$ and $C_{\mu_{B}}$ are positive. In particular, for the structure we have considered $1 / e^{2} N_{A(B)} \ll 1 / C_{A(B)}$; hence $C_{\mu_{A(B)}} \approx C_{A(B)}$. From this we deduce that the current correlations observed between the two gates in this system will be positive and characterized by Eq. (22).

Having considered the example where gate $B$ sees the same edge state as gate $A$, it is instructive to consider the case where gate $B$ is in position (ii). In this case a carrier 
TABLE I. Sign of equilibrium $\left(S_{I_{A} I_{B}}^{q}(\omega)\right)$ and nonequilibrium $\left(S_{I_{A} I_{B}}^{V}(\omega)\right)$ current correlations between gates $A$ and $B$ for the four positions of gate $B$ relative to gate $A$.

\begin{tabular}{lcccc}
\hline \hline & (i) & (ii) & (iii) & (iv) \\
\hline$S_{I_{A} I_{B}}^{q}(\omega)$ & $>0$ & $=0$ & $\geq 0$ & $\geq 0$ \\
$S_{I_{A} I_{B}}^{V}(\omega)$ & $\geq 0$ & $\leq 0$ & $=0$ & $=0$ \\
\hline \hline
\end{tabular}

which is seen by gate $A$ will not be seen by gate $B$. For this case the scattering matrix is as follows:

$$
\mathbf{S}=\left(\begin{array}{cc}
r e^{i \theta_{B}} & t e^{i \theta_{B}} \\
t^{\prime} e^{i \theta_{A}} & r^{\prime} e^{i \theta_{A}}
\end{array}\right)
$$

Following the same methodology as before we find

$$
\begin{gathered}
R_{q}^{A A}=R_{q}^{B B}=\left(h / 2 e^{2}\right), \\
R_{q}^{A B}=R_{q}^{B A}=0, \\
R_{V}^{A A}=R_{V}^{B B}=-R_{V}^{A B}=-R_{V}^{B A}=\left(h / e^{2}\right) T R .
\end{gathered}
$$

The equilibrium correlations proportional to $R_{q}^{A B}$ are zero, whereas the nonequilibrium correlations given by Eq. (26) are negative.

We also consider scenarios (iii) and (iv) depicted in Fig. 2. It is possible to calculate $R_{q}^{A B}$ and $R_{V}^{A B}$ which characterize the equilibrium and nonequilibrium current correlations between the two gates. For clarity we have summarized our results in Table I by showing the possible signs of current correlations between the two gates, for the four possible gate configurations shown in Fig. 2.

In this work we have shown that through the consideration of Coulomb coupling macroscopic gates to a mesoscopic conductor it is possible, for the example we have studied, to gain positive current correlations between the current fluctuations induced into the gates. The difference between this result and the result which would be obtained if we replaced our gates with leads, where the current correlations would be negative, can easily be understood by explaining the fundamental difference which arises when only Coulomb contacts are considered. This difference is that it is possible for a carrier to be seen by both contacts when the gates are attached only via the Coulomb interaction, which is not the case when we replace the gates with leads where a carrier cannot enter both leads. Consider scenario (i) shown in Fig. 2: a carrier which is seen by gate $A$ must also be seen by gate $B$. Replacing the gates with contacts, then a carrier traversing along the edge state of interest can go into either lead $A$ or lead $B$ or continue into lead 2.

We believe that this is the first prediction of such positive current correlations in normal mesoscopic conductors. Experimental work on induced charging in macroscopic gates Coulomb coupled to mesoscopic conductors has been carried out [16], and we suggest from this work that induced current fluctuations and correlations between gates in such systems should be examined in the future.

This work was supported by the Swiss National Science Foundation and the TMR network Dynamics of Nanostructures. We thank Ya. M. Blanter for useful discussions.

[1] R. Hanbury Brown and R. Q. Twiss, Nature (London) 177, 27 (1956).

[2] M. Büttiker, Phys. Rev. Lett. 65, 2901 (1990); Phys. Rev. B 46, 12485 (1992).

[3] Th. Martin and R. Landauer, Phys. Rev. B 45, 1742 (1992).

[4] Ya. M. Blanter and M. Büttiker, Phys. Rev. B 56, 2127 (1997); E. V. Sukhorukov and D. Loss, Phys. Rev. B 59, 13054 (1999); S. A. van Langen and M. Büttiker, Phys. Rev. B 56, R1680 (1997); Ya. M. Blanter and E. V. Sukhorukov, Phys. Rev. Lett. 84, 1280 (2000).

[5] For a broad review see Ya. M. Blanter and M. Büttiker, cond-mat/9910158 (unpublished).

[6] C. Texier and M. Büttiker, cond-mat/0003301 (unpublished), demonstrate that charge and current conserving inelastic scattering can lead to positive currentcorrelations.

[7] M. Henny et al., Science 284, 296 (1999).

[8] W. D. Oliver, J. Kim, R. C. Liu, and Y. Yamamoto, Science 284, 299 (1999).

[9] S. Oberholzer et al., Physica (Amsterdam) E 6, 314 (2000).

[10] Hybrid normal-superconducting systems should also show positive current correlations [S. Datta, P. F. Bagwell, and M. P. Anantram, Phys. Low-Dimens. Semicond. Struct. 3, 1 (1996); Th. Martin, Phys. Lett. A 220, 137 (1996)], due to Andreev scattering. However, recent work indicates that this effect can be observed only if the normal part is a single channel conductor [T. Gramespacher and M. Büttiker, Phys. Rev. B 61, 8125 (2000)]. See also Ref. [5].

[11] M. Büttiker, J. Math. Phys. (N.Y.) 37, 4793 (1996).

[12] M. H. Pedersen, S. A. van Langen, and M. Büttiker, Phys. Rev. B 57, 1838 (1998).

[13] F. T. Smith, Phys. Rev. 118, 349 (1960).

[14] M. Büttiker, cond-mat/9911188 (unpublished).

[15] M. Büttiker, Phys. Rev. B 41, 7906 (1990).

[16] W. Chen et al., Phys. Rev. Lett. 73, 146 (1994); P. K. H. Sommerfeld, R.W. van der Heijden, and F.M. Peeters, Phys. Rev. B 53, R13 250 (1996). 